\title{
A space fibre as added value in finite element modelling for optimal analysis of problems involving contact
}

\author{
Tewfik Ghomari ${ }^{\mathrm{a} *}$, Kamel Meftah $^{\mathrm{b}}$, Rezak Ayad ${ }^{\mathrm{c}}$ and Nabil Talbi ${ }^{\mathrm{d}}$ \\ ${ }^{a}$ Faculté de Mécanique, Université des Sciences et de la Technologie d'Oran (USTO), BP 1505, \\ El-Mnaouar, 31000 Oran, Algeria; ${ }^{b}$ Department of Mechanics, University of Biskra, 07000 Biskra, \\ Algeria; ${ }^{c}$ Laboratory of Engineering and Material Sciences, University of Reims Champagne-Ardenne, \\ ESIEC, Esp. Rolland Garros, BP 1029, F-51686 Reims, France; ${ }^{d}$ ANOTECH ENERGY, Bat Ampere, 6 \\ rue Ampere, 31670 Labege Cedex, France
}

\begin{abstract}
The present work deals with the non-linear formulation of an axisymmetric hyperelastic solid model, based on the concept space fibre rotation (SFR). The SFR-Axi model uses the kinematics of a space fibre to obtain a quite accurate displacement field. It improves in a significant way the precision of the linear element Q4-Axi. It can even be compared, on the accuracy and CPU time level, with the high-order elements as Q8-Axi for instance. A hyperelastic law, based on the Mooney-Rivlin model, is implemented to allow to the present model a better simulation of the forming process of hollow plastic bodies. The numerical results relate to primarily some known tests of hyperelastic structures, with and without contact (swellings).

Ce travail présente la formulation non-linéaire d'un modèle 3D axisymétrique basé sur le concept SFR (Space Fibre Rotation). Le modèle SFR-Axi utilise la cinématique d'une fibre dans l'espace pour obtenir un champ de déplacements enrichi. Il améliore la précision des éléments linéaires de type Q4-Axi. Ses résultats sont comparables, et même meilleurs en terme de temps CPU, à ceux des éléments d'ordre supérieur (Q8-Axi). Une loi constitutive hyperélastique de type Mooney-Rivlin est implémentée pour permettre au modèle développé de meilleures simulations appliquées à l'optimisation de procédés de formage de corps plastiques creux. Les résultats numériques assez prometteurs concernent essentiellement des problèmes tests de structures hyperélastiques connus de la littérature, avec et sans contact.
\end{abstract}

Keywords: special finite elements; contact; hyperelasticity; space fibre rotation

Mots-clés: éléments finis spéciaux; contact; hyperélasticité; space fibre rotation

\section{Introduction}

The plastics industry often uses simulation tools to reduce study time, and reduce the number of expensive tests experiment. These tools are also used to optimise the process parameters of certain (given) technology known as thermoforming and blow moulding hollow plastic. This technology has the disadvantage, in the step of blowing the hot parison becomes rigid when it touches the cold walls of the mould, and this collage is stretching up to touch the bowl full of all walls. The consequence of this process is a product with an irregular distribution of

*Corresponding author. Email: tewfikghomari@yahoo.com 
thicknesses. To solve this problem, numerical simulation can be used to predict areas where the thicknesses are thinner and the value of the blowing pressure to be applied (Wang \& Makinouchi, 2000). Modelling the complex behaviour of this type of equipment requires advanced mathematical and numerical tools (Crisfield, 1997; Mooney, 1940; Ogden, 1972). However, to realise a digital simulation of processes such as thermoforming or blow, we have to overcome several difficulties. The most annoying is the incompressibility of the digital element and large deformations of such materials hyperelastic or viscoelastic.

The management of contact between the parison and the mould is described in Heinstein, Mello, Attaway, and Laursen (2000) and Zhong and Nilsson (1994). The calculation of the reactions which is calculated either by the penalty method or by the Lagrange multipliers method also has a major difficulty to overcome. The approach widely used to simulate this type of material is known as Mooney-Rivlin model (Mooney, 1940; Rivlin, 1948). A simplified model, introduced by Williams (1970) and Treloar (1976) also called Neo-Hooke model, for which the function of the strain energy density is expressed in terms of tensor invariants of the CauchyGreen right. Another possibility is to use the model of Ogden (1972) with a density of strain energy which is formulated in terms of principal stretches. Note that the Ogden model is considered as one of the most complex constitutive laws used in numerical modelling.

The main contribution of this work comes from the observation that the current finite element formulations for hyperelastic materials were unsatisfactory (Sze, Zhenga, \& Lob, 2004), especially when using shell elements in large strain. Most efforts have been focused on elements of the membrane, which have the advantage of having a plane stress behaviour. This eliminates the problem of incompressibility in finite element formulation. Volumetric 3D elements (3D or 3D axisymmetric) have numerical difficulties due to the incompressibility (hydrostatic pressure) and require a penalty of strain energy density (Crisfield, 1997; Ziane, 1999). Recently, several studies have focused on the development of finite elements. Sze has developed an 18-node hyperelastic solid with a stabilisation technique to eliminate the membrane locking and shear.

In this work, a new 3D axisymmetrical four-node element, labelled Space Fibre Rotation Quadrilateral Axisymmetric (SFRQ-Axi), has been developed for axisymmetric problems using the Mooney-Rivlin hyperelastic model. It is based on the "SFR" concept (Ayad, 2002; Zouari, Ayad, Ben Zineb, \& Benjeddou, 2012) which allows additional rotation degrees of freedom (dof) in order to give the element a high accuracy without increasing the number of element nodes.

Two well-known tests are presented. They show the good performances of the present element in terms of accuracy and computational time, when compared to the eight-node quadratic element Q8-Axi for instance.

\section{Formulation of the special element model SFRQ-Axi}

The implementation of a hyperelastic law in the case of thin structures, with the assumption of plane stress hypothesis, makes it possible to deal directly with the problem of incompressibility $(\mathrm{I} 3=1)$ and to determine the hydrostatic pressure $p$. In 3D solid or 3D axisymmetric formulations, the pressure cannot be determined by the boundary conditions. To overcome this difficulty, we adopt a quasi-incompressible formulation suggested by Crisfield. It consists of uncoupling the strain energy in a volumetric energy, related to dilation, and the energy of distortion, related to the isochoric deformation.

We present the main equations used for the implementation of the behaviour law in an axisymmetric solid element. A total Lagrangian formulation associated with a displacement 
model using the concept of "SFR", is used. The final model is a four-node 3D axisymmetric finite element with three dof per node. Only $2 \times 2$ Gauss points are used.

\subsection{Geometry and kinematic aspects}

The position vector of a point $p$ in the meridian plan, expressed in a cylindrical base, is given by:

$$
\vec{x}_{p}=r \vec{i}_{r}+z \vec{k} \quad \text { with } \quad r=\sum_{i=1}^{4} N_{i} r_{i} ; \quad z=\sum_{i=1}^{4} N_{i} z_{i}
$$

The approximation of the displacement field of point $p(r, Z)$ of the plan will take into account the space rotation of the fibre ip (Figure 1). It takes the following form:

$$
\begin{gathered}
\left\{\begin{array}{c}
U \\
W
\end{array}\right\}=\sum_{i=1}^{4} N_{i}\left\{\begin{array}{c}
U_{i} \\
W_{i}
\end{array}\right\}+\sum_{i=1}^{4} N_{i} \vec{\theta}_{y_{i}} \wedge\left(\vec{r}-\vec{r}_{i}\right) \\
\vec{\theta}_{x_{i}}=\theta_{x_{i}} \vec{j} \quad \text { and } \quad i=1 \ldots 4
\end{gathered}
$$

where

$$
\vec{\theta}_{y_{i}} \wedge\left(\vec{r}-\vec{r}_{i}\right)=\left\{\begin{array}{c}
-\theta_{y_{i}}\left(z-z_{i}\right) \\
\theta_{y_{i}}\left(r-r_{i}\right) \\
0
\end{array}\right\}
$$

$\vec{j}$ is the unit vector following the axis $y$ perpendicular to plan $(r, o, z)$, whereas $\left(\vec{r}-\vec{r}_{i}\right)$ is the position vector of the fibre connecting the point $p$ to the node $i$ (Figure 1). The fibre rotations $\theta_{y}$ are only defined on the element top nodes.

The final expression of components $\langle u, w\rangle$ is written in a matrix form as:

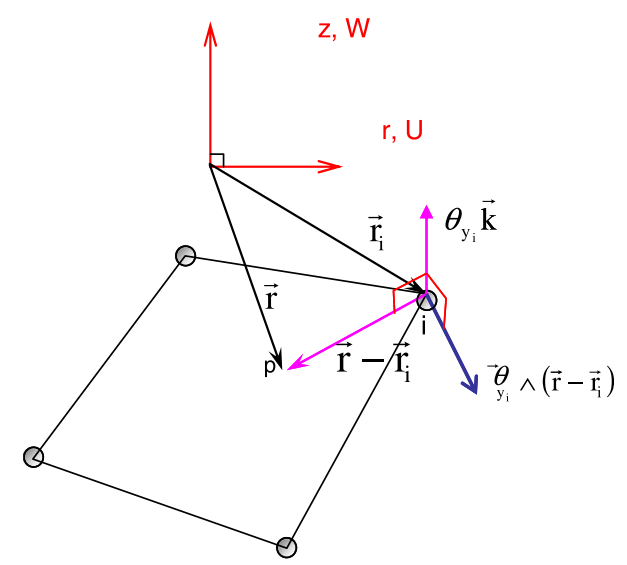

Figure 1. Kinematics of the virtual fibre ip. 


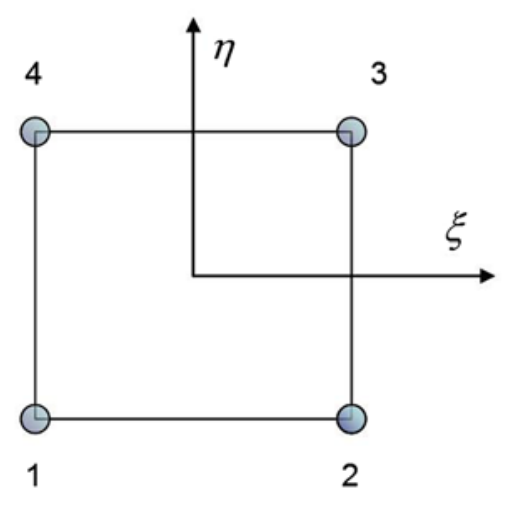

Reference element

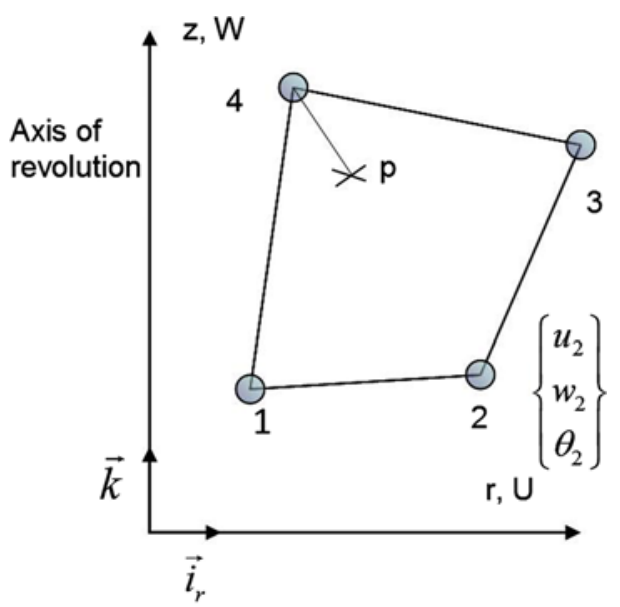

Real element

Figure 2. Axisymmetric element SFRQ-Axi.

$$
\begin{gathered}
\left\{\begin{array}{c}
U \\
W
\end{array}\right\}=\left[\begin{array}{ccc}
N_{i} & 0 & -N_{i}\left(z-z_{i}\right) \\
0 & N_{i} & N_{i}\left(r-r_{i}\right)
\end{array}\right]\left\{U_{n}\right\} \quad \text { with } i=1 \ldots 4 \\
\text { and }\left\langle U_{n}\right\rangle=\left\langle\cdots u_{i} w_{i} \theta_{z i} \cdots i=1 \ldots 4\right\rangle
\end{gathered}
$$

The shape functions $N_{i}$ are those of the classical four-node bilinear element Q4 (Figure 2):

$$
\begin{array}{ll}
N_{1}=\frac{(1-\xi)(1-\eta)}{4} & N_{2}=\frac{(1+\xi)(1-\eta)}{4} \\
N_{3}=\frac{(1+\xi)(1+\eta)}{4} & N_{4}=\frac{(1-\xi)(1+\eta)}{4}
\end{array}
$$

\subsection{Strain tensor}

The strain gradient tensor $[F]$ is classically given for the axisymmetric case by the following expression

$$
[F]=\left[\begin{array}{ccc}
1+U, r^{0} & 0 & U, z^{0} \\
0 & 1+\frac{U}{r^{0}} & 0 \\
W, r^{0} & 0 & 1+W_{, z^{0}}
\end{array}\right]
$$

The Right Cauchy-Green tensor $C=F^{\mathrm{T}} F$ in cylindrical base becomes

$$
[C]=\left[\begin{array}{ccc}
\left(1+U_{, r}\right)^{2}+W_{, r}^{2} & 0 & \left(1+U_{, r}\right) U_{, z}+\left(1+W_{, z}\right) W_{, r} \\
0 & \left(1+\frac{U}{r}\right)^{2} & 0 \\
\left(1+U_{, r}\right) U_{, z}+\left(1+W_{, z}\right) W_{, r} & 0 & U_{, r}^{2}+\left(1+W_{, z}\right)^{2}
\end{array}\right]
$$


Three known invariants of tensor [C] may be deducted:

$$
\begin{aligned}
& I_{1}=C_{11}+C_{22}+C_{33} \\
& I_{2}=C_{11} C_{22}+C_{22} C_{33}+C_{33} C_{11}-C_{13}^{2} \\
& I_{3}=C_{11} C_{22} C_{33}-C_{22} C_{13}^{2}
\end{aligned}
$$

\subsection{Principle of virtual work}

The total potential energy is defined by Equation (9) for sum of difference k internal potential energy elementary (10) and external energy elementary:

$$
\Pi=\sum_{k}\left(\Pi_{\mathrm{int}}^{k}-\Pi_{\mathrm{ext}}^{k}\right)
$$

With

$$
\Pi_{\text {int }}^{k}=\int_{v^{0}} W\left(\bar{I}_{1}, \bar{I}_{2}, J\right) \mathrm{d} v^{0}
$$

$W$ is the strain energy per unit of no deformed volume accumulated in the structure during the deformation. It is defined by Crisfield (1997).

In general, we work with the compressible form of Mooney-Rivlin law this relation has the drawback of being zero if the principal stretches are equal. To overcome this difficulty, we may add a compressible term of the bulk modulus $K$. Hence, using these modified invariants, with $W_{\mathrm{b}}$ (11a) and adapting $W_{\mathrm{d}}$ (deviatoric) from (11b), the modified Mooney-Rivlin function might involve

$$
\begin{gathered}
W_{\mathrm{b}}=\frac{1}{2} \kappa(J-1)^{2} \\
W_{\mathrm{d}}=C_{1}\left(\bar{I}_{1}-3\right)+C_{2}\left(\bar{I}_{2}-3\right) \\
W\left(\bar{I}_{1}, \bar{I}_{2}, J\right)=W_{\mathrm{d}}\left(\bar{I}_{1}, \bar{I}_{2}\right)+W_{\mathrm{b}}(J) \\
W\left(\bar{I}_{1}, \bar{I}_{2}, J\right)=C_{1}\left(\bar{I}_{1}-3\right)+C_{2}\left(\bar{I}_{2}-3\right)+\frac{1}{2} \kappa(J-1)^{2}
\end{gathered}
$$

where $\bar{I}_{1}$ and $\bar{I}_{2}$ represent the modified invariants. The following corresponding expressions are given by Crisfield (1997):

$$
\bar{I}_{1}=I_{1} I_{3}^{-\frac{1}{3}}=I_{1} J^{-\frac{2}{3}} ; \quad \bar{I}_{2}=I_{2} I_{3}^{-\frac{2}{3}}=I_{2} J^{-\frac{4}{3}} ; \quad J=I_{3}^{\frac{1}{2}} ; \quad \kappa=\frac{E}{3(1-2 v)}
$$

The variation of the external potential energy $\Pi_{\mathrm{ext}}^{k}$ is given by: 


$$
\delta \Pi_{\mathrm{ext}}^{k}=\iint_{S^{0}}\langle\delta u\rangle\{p\} \mathrm{d} S^{0}
$$

$\langle\delta u\rangle$ is the displacement in the local base and $\{p\}$ is the compressive forces distributed along the meridian line. The first variation of the total energy is given by

$$
\delta \Pi=\sum_{k}\left(\delta \Pi_{\mathrm{int}}^{k}-\delta \Pi_{\mathrm{ext}}^{k}\right)=0
$$

where $\delta \Pi_{\text {int }}^{k}$ represents the first variation of internal elementary energy:

$$
\delta \Pi_{\text {int }}^{k}=\int_{V^{0}} \delta W\left(\bar{I}_{1}, \bar{I}_{2}, J\right) \mathrm{d} V^{0}
$$

\subsection{Residual vector}

The expression of the residual vector $\left\{f_{\text {int }}^{k}\right\}$ is given from those of $\delta \Pi_{\text {int }}^{k}(17)$ :

$$
\begin{aligned}
& \delta \Pi_{\text {int }}^{k}=2 \pi\left\langle\delta U_{1} \quad \delta W_{1} \quad \delta \theta_{1} \quad \cdots \quad \delta U_{4} \quad \delta W_{4} \quad \delta \theta_{4}\right\rangle \int_{\xi} \int_{\eta}\{\bar{r}\} r^{0} \operatorname{det} J d \xi d \eta \\
& \delta \Pi_{\text {int }}^{k}=\langle\delta U\rangle\left\{f_{\text {int }}^{k}\right\}
\end{aligned}
$$

Using the Mooney-Rivlin law, $\delta \Pi_{\text {int }}^{k}$ becomes

$$
\delta \Pi_{\mathrm{int}}^{k}=\int_{V^{0}}\left(A_{1} \delta \bar{I}_{1}+A_{2} \delta \bar{I}_{2}+A_{3} \delta \bar{I}_{3}\right) d V^{0}
$$

With

$$
A_{1}=C_{1} I_{3}^{-\frac{1}{3}} ; \quad A_{2}=C_{2} I_{3}^{-\frac{2}{3}} ; \quad A 3=-\frac{1}{3} C_{1} I_{1} I_{3}^{-\frac{4}{3}}-\frac{2}{3} C_{2} I_{2} I_{3}^{-\frac{5}{3}}+\frac{1}{2} \frac{\kappa(J-1)}{J}
$$

The modified three invariants $\bar{I}_{1}, \bar{I}_{2}, \bar{I}_{3}$ of tensor [C] (7) appear under their first variation $\delta \bar{I}_{1}, \delta \bar{I}_{2}$ and $\delta \bar{I}_{3}$ :

$$
\begin{aligned}
& \delta \bar{I}_{1}=\operatorname{tr}(\delta[C])=\delta C_{11}+\delta C_{22}+\delta C_{33} \\
& \delta \bar{I}_{2}=\left(C_{22}+C_{33}\right) \delta C_{11}+\left(C_{11}+C_{33}\right) \delta C_{22}+\left(C_{11}+C_{22}\right) \delta C_{33}-2 C_{13} \delta C_{13} \\
& \delta \bar{I}_{3}=C_{22} C_{33} \delta C_{11}+\left(C_{11} C_{33}-C_{13}^{2}\right) \delta C_{22}+C_{11} C_{22} \delta C_{33}-2 C_{22} C_{13} \delta C_{13}
\end{aligned}
$$

Substituting Equation (21) leads to the following expression of the elementary internal strain potential:

$$
\delta \Pi_{\text {int }}^{k}=\int_{V^{0}} T_{\mathrm{a}} \delta U,_{r}+T_{\mathrm{b}} \delta U_{, z}+T_{\mathrm{c}} \delta W_{, r}+T_{\mathrm{d}} \delta W_{, z}+T_{\mathrm{e}} \delta U d V^{0}
$$




$$
\begin{array}{ll}
T_{\mathrm{a}}=2 B_{1}\left(1+U,_{r}\right)+B 4 U_{, z} ; & T_{\mathrm{b}}=2 B_{3} U_{, z}+B_{4}\left(1+U_{, r}\right) \\
T_{\mathrm{c}}=2 B_{1} W_{, r}+B_{4}\left(1+W,_{z}\right) ; & T_{\mathrm{d}}=2 B_{3}\left(1+W,_{z}\right)+B_{4} W_{, r} ; \quad T_{\mathrm{e}}=\frac{2 B_{2}}{r^{0}}\left(1+\frac{U}{r^{0}}\right)
\end{array}
$$

where the expressions $B_{1}, B_{2}, B_{3}, B_{4}$ are given by

$$
\begin{array}{ll}
B_{1}=A_{1}+\left(C_{22}+C_{33}\right) A_{2}+C_{22} C_{33} A_{3} ; & B_{2}=A_{1}+\left(C_{11}+C_{33}\right) A_{2}+\left(C_{11} C_{33}-C_{13}^{2}\right) A_{3} \\
B_{3}=A_{1}+\left(C_{11}+C_{22}\right) A_{2}+C_{11} C_{22} A_{3} ; & B_{4}=-2\left(C_{13} A_{2}+C_{22} C_{13} A_{3}\right)
\end{array}
$$

For the expression of internal residual vector, we need to define the first variation of the Right Cauchy-Green tensor $\delta C$ :

$$
\begin{aligned}
& \delta C_{11}=2\left(\left(1+U_{, r}\right) \delta U_{, r}+W_{, r} \delta W_{, r}\right) ; \quad \delta C_{22}=2\left(1+\frac{U}{r^{0}}\right) \frac{\delta U}{r^{0}} \\
& \delta C_{33}=2\left(\left(1+W_{, z}\right) \delta W_{, z}+U_{, z} \delta U_{, z}\right) \\
& \delta C_{13}=U_{, z} \delta U_{, r}+\left(1+W_{, z}\right) \delta W+_{, z}\left(1+U_{, r}\right) \delta U_{, z}+W_{, r} \delta W_{, z}
\end{aligned}
$$

The variation of the internal strain energy becomes

$$
\delta \Pi_{\mathrm{int}}^{k}=\int_{V^{0}}\left(Z_{a} \delta U_{i}+Z_{b} \delta W_{i}+Z_{c} \delta \theta_{y i}\right) d V^{0}
$$

where $Z_{a}, Z_{b}$ and $Z_{c}$ are given by

$$
\begin{aligned}
& Z_{a}=B_{1} E_{1}+B_{2} E_{2}+B_{3} E_{3}+B_{4} E_{4} \\
& Z_{b}=B_{1} E_{5}+B_{3} E_{6}+B_{4} E_{7} \\
& Z_{c}=B_{1} E_{8}+B_{2} E_{9}+B_{3} E_{10}+B_{4} E_{11}
\end{aligned}
$$

$$
\begin{aligned}
& E_{1}=2(1+U, r) N_{i, r} ; \quad E_{2}=\frac{2}{r}\left(1+\frac{U}{r}\right) N_{i} ; \quad E_{3}=2 U_{, z} N_{i, z} \\
& E_{4}=(1+U, r) N_{i, z}+U_{, z} N_{i, r} ; \quad E_{5}=2 W_{, r} N_{i, r} ; \quad E_{6}=2\left(1+W,_{, z}\right) N_{i, z} \\
& E_{7}=\left(1+W_{, z}\right) N_{i, r}+W_{, r} N_{i, z} ; \quad E_{8}=2\left(1+U_{, r}\right) N_{i, r}\left(-z+z_{i}\right)+2 W,_{, r}\left(N_{i, r}\left(r-r_{i}\right)+N_{i}\right) \\
& E_{9}=-\frac{2}{r}\left(1+\frac{U}{r}\right) N_{i}\left(z-z_{i}\right) ; \quad E_{10}=2\left(1+W_{, z}\right) N_{i, z}\left(r-r_{i}\right)+2 U_{, z}\left(N_{i, z}\left(-z+z_{i}\right)-N_{i}\right) \\
& E_{11}=\left(U_{, z} N_{i, r}\left(-z+z_{i}\right)\right)+\left(1+W_{, z}\right)\left(N_{i, r}\left(r-r_{i}\right)+N_{i}\right)+W_{, r} N_{i, z}\left(r-r_{i}\right)+(1+U, r)\left(N_{i, z}\left(-z+z_{i}\right)-N_{i}\right)
\end{aligned}
$$

And

$$
\begin{gathered}
\mathrm{d} V^{0}=2 \pi r^{0} \operatorname{det} J \mathrm{~d} \xi \mathrm{d} \eta \\
\left\langle N_{i, r}\right\rangle=j_{11}\left\langle N_{i, \xi}\right\rangle+j_{12}\left\langle\begin{array}{l}
\left.N_{i, \eta}\right\rangle \\
\left\langle N_{i, z}\right\rangle=j_{21}\left\langle N_{i, \xi}\right\rangle+j_{22}\left\langle N_{i, \eta}\right\rangle
\end{array} \quad \begin{array}{c}
j_{k l} \quad k, l=1 \cdots 2 \\
\text { Terms of Jacobian matrix }
\end{array}\right.
\end{gathered}
$$

The final expression of the internal force vector can be easily derived from $\delta \Pi_{\text {int }}^{k}(25)$ with a $2 \times 2$ Gauss point schema for computing the integral terms 


$$
\delta \Pi_{\text {int }}^{k}=\langle\delta U\rangle\left\{f_{\text {int }}^{k}\right\}
$$

\section{Polymer-mould contact algorithm}

In this section, we present a contact technique with the corresponding search algorithm, used for modelling problems involving contact. The algorithm of total research is based on the work of Heinstein et al. (2000). We have developed in our Ph.D. thesis (Ghomari, 2007) a local research algorithm, using penalty method, that we will detail in the following sections. There are other techniques of research of the contact zones, which make it possible to avoid a systematic sweeping of the whole of the nodes to each time step. The principle rests on a space division using a virtual mesh. Lists of objects containing the nodes in each zone are updated at each new introduction of object. The simplest structure is the regular mesh covering the field. Each box of mesh is indexed by an entirety. This structure is described in Figure 3.

In order to cure the need for locating the nodes candidates in contact with an arbitrary manner, a total strategy is adopted which consists of the following.

Initialise the vector (nbox) which contains the numbers of the nodes in each bucket of a virtual mesh (Figure 3).

- Find the code Bi for each node.

- Store the codes of all nodes $i$ in a vector (lbox): lbox $(I)=\mathrm{Bi}$.

- Increment the storyteller for the codes of such kind: nbox $(\mathrm{Bi})=\mathrm{nbox}(\mathrm{Bi})+1$.

- Calculate the pointer of each code $j$ in a list of nodes.

- Npoint $(1)=1$, npoint $(j)=\operatorname{npoint}(j-1)+\operatorname{nbox}(j-1)$.

- Initialise nbox with zero.

- Find the nodes slaves in agreement with the numbers of code in a list (ndsort).

- Ndsort $(\operatorname{nbox}(\operatorname{lbox}(I))+\operatorname{npoint}(\operatorname{lbox}(I)))=\operatorname{Inbox}(\operatorname{lbox}(I))=\operatorname{nbox}(\operatorname{lbox}(I))+1$.

The code of each bucket is determined by the expression below:

$$
B_{i}=\left(S_{Y}^{i}-1\right) S_{X}+S_{X}^{i}
$$

with

$$
\begin{aligned}
& S_{X}=\operatorname{int}\left[\left(x_{\max }-x_{\min }\right) / b_{\mathrm{s}}\right]+1 \\
& S_{Y}=\operatorname{int}\left[\left(y_{\max }-y_{\min }\right) / b_{\mathrm{s}}\right]+1
\end{aligned}
$$
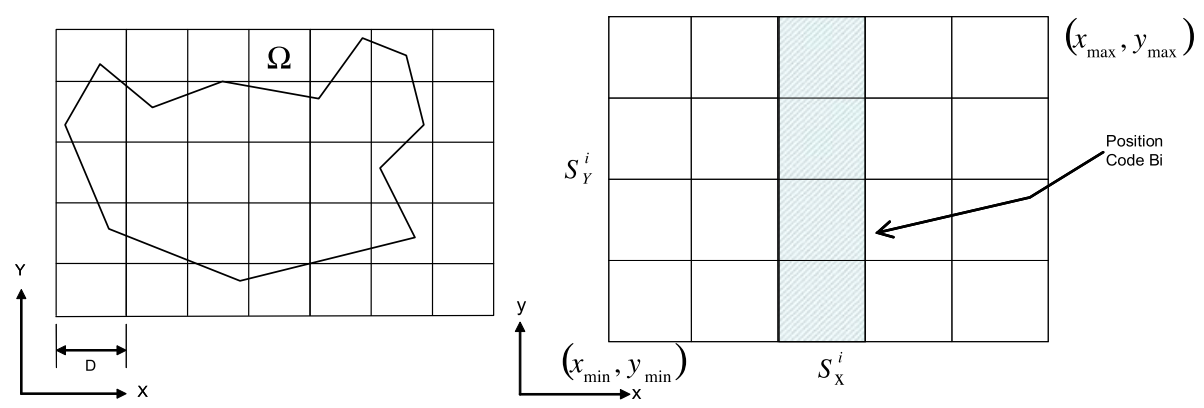

Figure 3. The position codes buckets according to the node containing $S_{x y}^{i}$. 
where, $b_{\mathrm{s}}$ is the width of bucket.

The cutting which contains any node is calculated as follows:

$$
\begin{aligned}
& S_{X}^{i}=\operatorname{int}\left[\left(x_{i}-x_{\min }\right) / b_{\mathrm{s}}\right]+1 \\
& S_{Y}^{i}=\operatorname{int}\left[\left(y_{i}-x_{\min }\right) / b_{\mathrm{s}}\right]+1
\end{aligned}
$$

The collection of the slaves nodes with their potential main segment is given as follows. Construction of a territory widened for each main surface (Figure 4).

Determination of the codes of the nodes of main segments in the widened territory.

$$
\begin{aligned}
& \text { ibox }_{\min }=\min \left(S_{X}, \operatorname{int}\left(\left(x c_{\min }-x_{\min }\right) / b_{s}\right)+1\right) \\
& \text { jbox }_{\min }=\min \left(S_{Y}, \operatorname{int}\left(\left(y c_{\min }-y_{\min }\right) / b_{s}\right)+1\right) \\
& \text { ibox }_{\max }=\min \left(S_{X}, \operatorname{int}\left(\left(x c_{\max }-x_{\min }\right) / b_{s}\right)+1\right) \\
& \text { jbox }_{\max }=\min \left(S_{Y}, \operatorname{int}\left(\left(y c_{\max }-y_{\min }\right) / b_{s}\right)+1\right)
\end{aligned}
$$

where $x c_{\min }$ and $x c_{\max }$ are the ends of buckets widened in the direction of $x$, just as $y c_{\min }$, $y c_{\max }$ in the directions of $y$.

Identification of the code of each bucket widened while determining arranged in two directions $X$ and $Y$.

$$
\begin{aligned}
& \text { Loop from ibox }=i b o x_{\min } \text { to } i b o x_{\max } \\
& \text { Loop from jbox }=j b o x_{\min } \text { to } j b o x_{\max } \\
& B_{i}=(j b o x-1) S_{X}+i b o x
\end{aligned}
$$

\section{End Loop}

\section{End Loop}

All the codes of the nodes calculated are potentially able to interact with the main segment.

\subsection{Binary sort}

The binary sort is to sort the coordinates of the nodes along the two directions $(x, y)$ by sorting algorithms very efficient operations of the order $\mathrm{O}\left(\mathrm{Nlog}_{2} N\right)$ of permutations. This establishes a

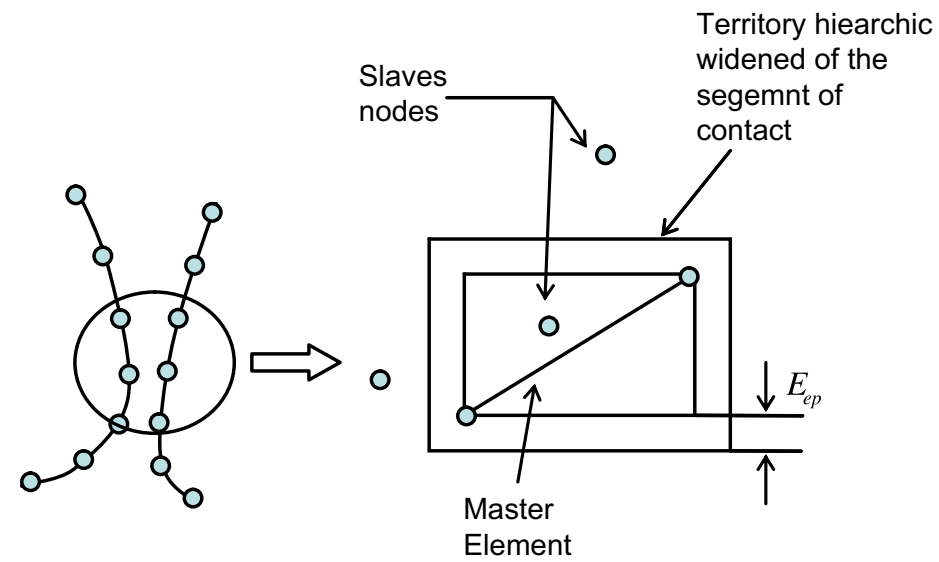

Figure 4. Definition of the hierarchical widened territory of a main element. 
database of vectors containing the numbers of nodes, and two other vectors that contain pointers to nodes in sorting functions previously performed. For each master surface, it lists the nodes identified by the search algorithm within the expanded territory of the boxes below ( $x$, $y$ ). Once this task is completed, we determine the vector that stores fewer nodes of the two sorted vectors. This step minimises the exhaustive search over all directions simultaneously on all nodes of the structures, which restricts the global search for candidates boxes containing nodes contacts. This strategy is adopted in our study in order to locate contact cases relatively difficult (see Figure 5). The difficulty of the problem lies in the fact that nodes 2 and 14 may have the same code box and become candidates for the contact. These two nodes can cross the master surface $8-9$ without being detected by the algorithm.

At the research level of local contact, we have developed in this study, an algorithm to detect the slave nodes that can cross the segment master (Figure 6).

\subsection{Discretisations}

The local reference mark is defined on the element (1-2) (Figure 7) by the base $(\vec{t}, \vec{n})$ with $\vec{t}$ tangent vector whose components in the total reference mark with two dimensions are:

$$
\{t\}=\frac{1}{L}\left(\left\{X_{2}\right\}-\left\{X_{1}\right\}\right)=\left\{\begin{array}{l}
c \\
s
\end{array}\right\}
$$

And $\vec{n}$ the normal vector such as:

$$
\{n\}=\left\{\begin{array}{c}
-s \\
c
\end{array}\right\} \quad \text { with } \quad L=\left\|X_{2}-X_{1}\right\| \begin{gathered}
c: \cos \text { inus } \\
s: \text { Sinus }
\end{gathered}
$$

That is to say $\xi$ the standardised curvilinear coordinate of $p$ (the projection of $S$ on the segment (1-2)) for the case two-dimensional:

$$
\{\xi\}=\frac{1}{L}\left\langle X_{S}-X_{1}\right\rangle\{t\} \quad \text { with } \quad 0 \leq \xi \leq 1
$$

The normal gap is the distance from penetration of the node $S$ through the element (1-2) or a surface is expressed in the local reference mark and the tangential gap is defined by

$$
\left\{x_{n}\right\}=\left\langle X_{S}-X_{1}\right\rangle\{n\} ; \quad\left\{x_{t}\right\}=\left\langle X^{s}-X^{*}\right\rangle\{t\}
$$

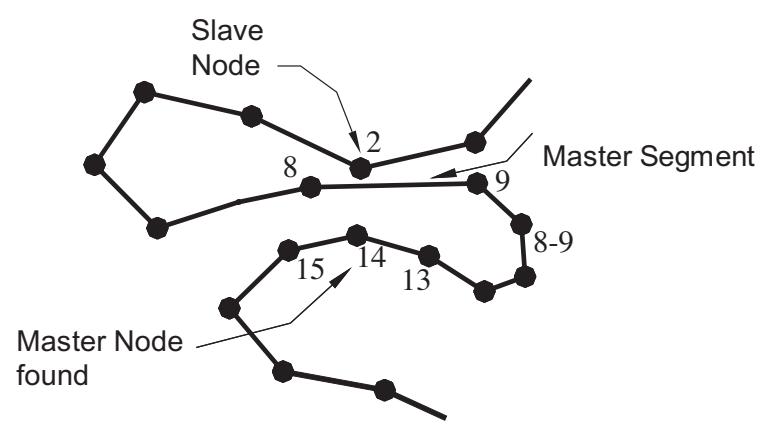

Figure 5. Difficult case of contact problem. 


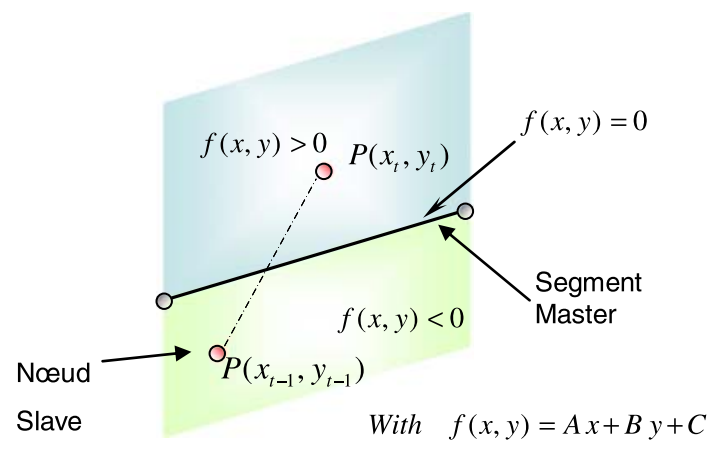

Figure 6. Detection of the passage of the node.

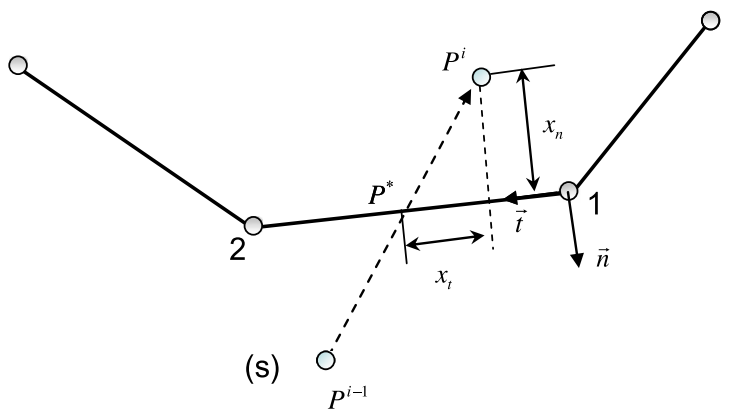

Figure 7. Local state 2D.

At the time of the resolution of the problem of balance by a schema of Newton-Raphson, an analysis of the positions of the nodes candidates to the contact is carried out with each iteration. The statute of the nodes is evaluated for the calculation of the reactions by the law of Coulomb and is given by the following expressions:

Normal reaction:

$$
\begin{aligned}
& \text { If } \quad x_{n} \geq 0 \quad \text { no contact } \Rightarrow r_{n}=0 \\
& \text { If } \quad x_{n}<0 \quad \text { contact } \Rightarrow r_{n}=k_{n} x_{n}
\end{aligned}
$$

Tangential reaction:

$$
\begin{gathered}
r_{t_{\mathrm{cri}}}=\mu r_{n} \quad \text { critical reaction } \\
r_{t}=k_{t} x_{t} \quad \text { tangential reaction }
\end{gathered}
$$

where $\mu$ represents the coefficient of friction

$$
\text { If }\left|r_{t}\right|<r_{t_{\mathrm{cri}}} \text { adhering contact } \Rightarrow r_{t}=k_{t} x_{t}
$$



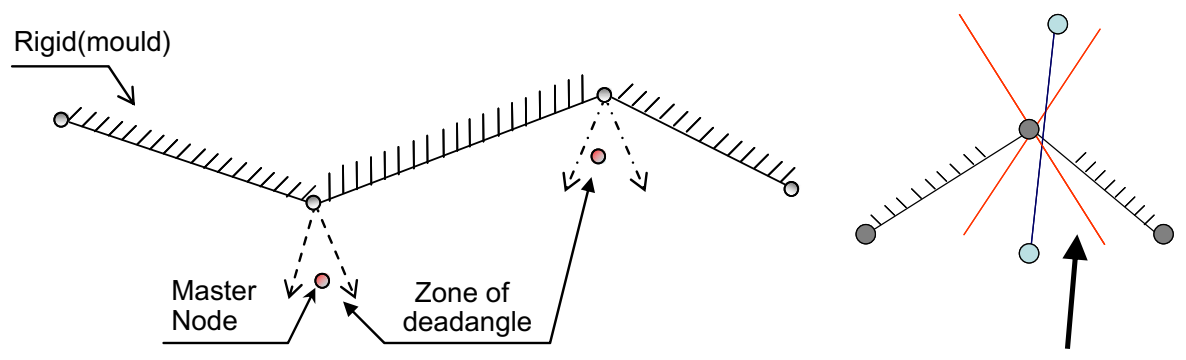

Figure 8. Ambiguous cases 2D trajectories met.

$$
\text { If } \quad\left|r_{t}\right| \geq r_{t_{\mathrm{cri}}} \quad \text { slipping contact } \Rightarrow r_{t}=r_{t_{\mathrm{cri}}} \frac{x_{t}}{\left|x_{t}\right|}
$$

The algorithm of search for contact must be able to manage ambiguous situations, which can occur at the time of the procedure of search for contact (see Figure 8):

\section{Numerical experiments}

All our geometry and results have been drawn and visualised using the educational version of GID software (www.gidhome.com).

\subsection{Circular plate under uniform pressure}

We consider a circular plate treated by Hughes and Carnoy (1983). Radius $L$ and thickness $h$ are, respectively, 7.5 in $(19.05 \mathrm{~cm})$ and 0.5 in $(1.27 \mathrm{~cm})$. The plate is fixed to its circumference and is subjected to a pressure on one of its faces (Figure 9). We use a quasi-incompressible hyperelastic material represented by the Mooney-Rivlin's model with the constants $C_{1}=80 \mathrm{psi}(0.55 \mathrm{MPa})$ and $C_{2}=20 \mathrm{psi}(0.14 \mathrm{MPa})$. The penalty factor $\kappa$ used to penalise the internal energy is taken equal to $10^{4}(68.9 \mathrm{MPa})$. The penalty coefficient of the energy is chosen as large as possible to attenuate the effect of incompressibility so that calculations of the simulation does not block.

This test is solved in quasi-static. Various meshes were used to analyse the performance of SFRQ-Axi element in terms of CPU time, comparatively with the Q4-Axi element and the Q8-Axi element taken as a reference solution. The loading curve pressure is obtained by controlling the extreme node of the plate. Four different meshes are tested (Figure 10). The last mesh, named "same" in Figure 12, allows getting the same number of dof (ddl) for the three elements: the problem is resolved using the same size of memory.

Figure 11 shows that the results of the pressure variation, in accordance with the vertical displacement of the middle point of the plate, are almost identical compared to those given

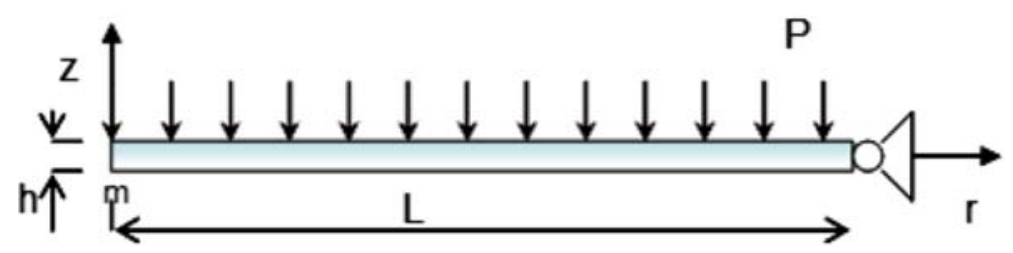

Figure 9. Circular plate under pressure. 


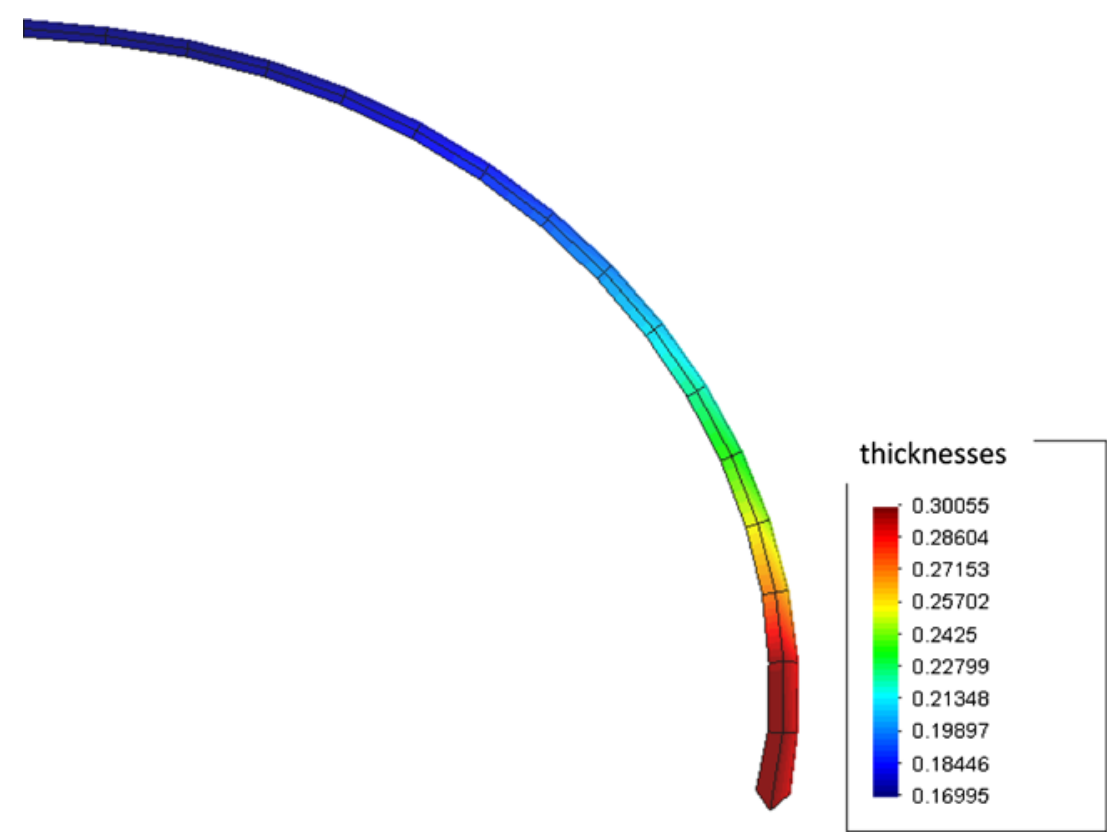

Figure 10. Distribution of thicknesses (in).

(in)

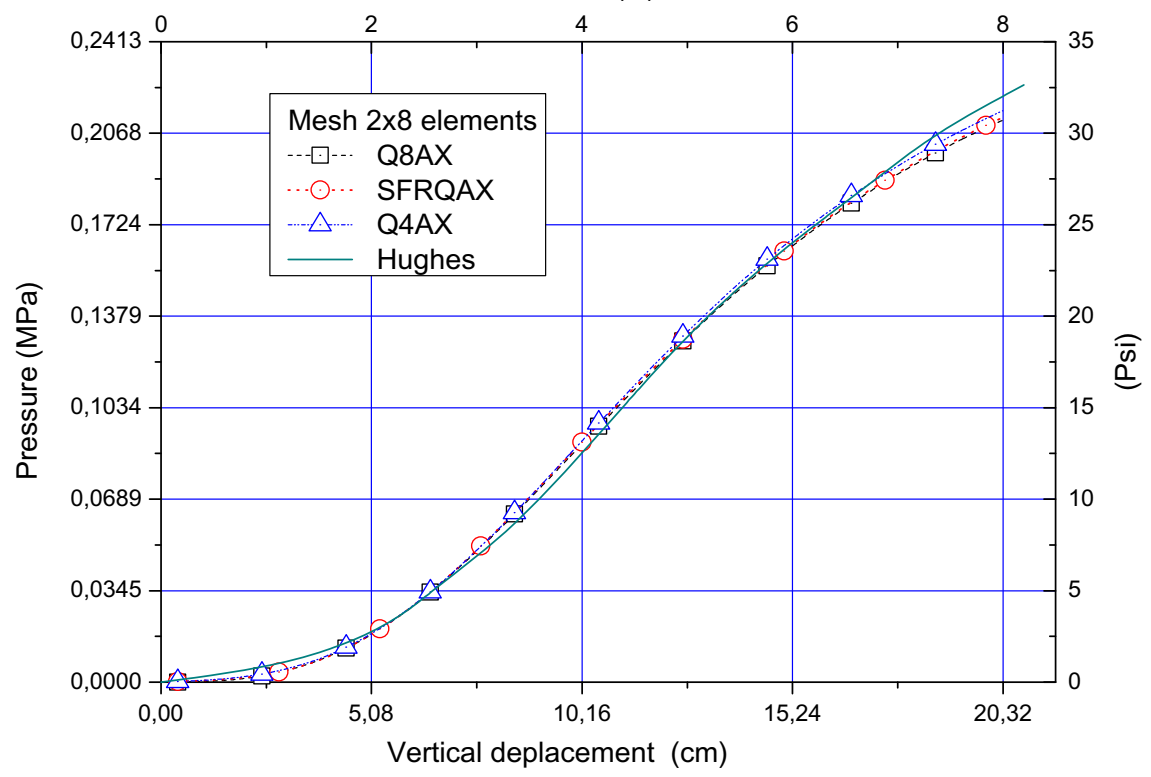

Figure 11. Loading curve.

by Hughes and Carnoy (1983). For a given strain step, we have represented in Figure 10 an image of the thickness distribution, showing the advantage use 3D axisymmetric elements. Compared to the results of the quadratic element Q8-Axi in Figure 11, the element SFR-Axi 
Time CPU for various Mesh

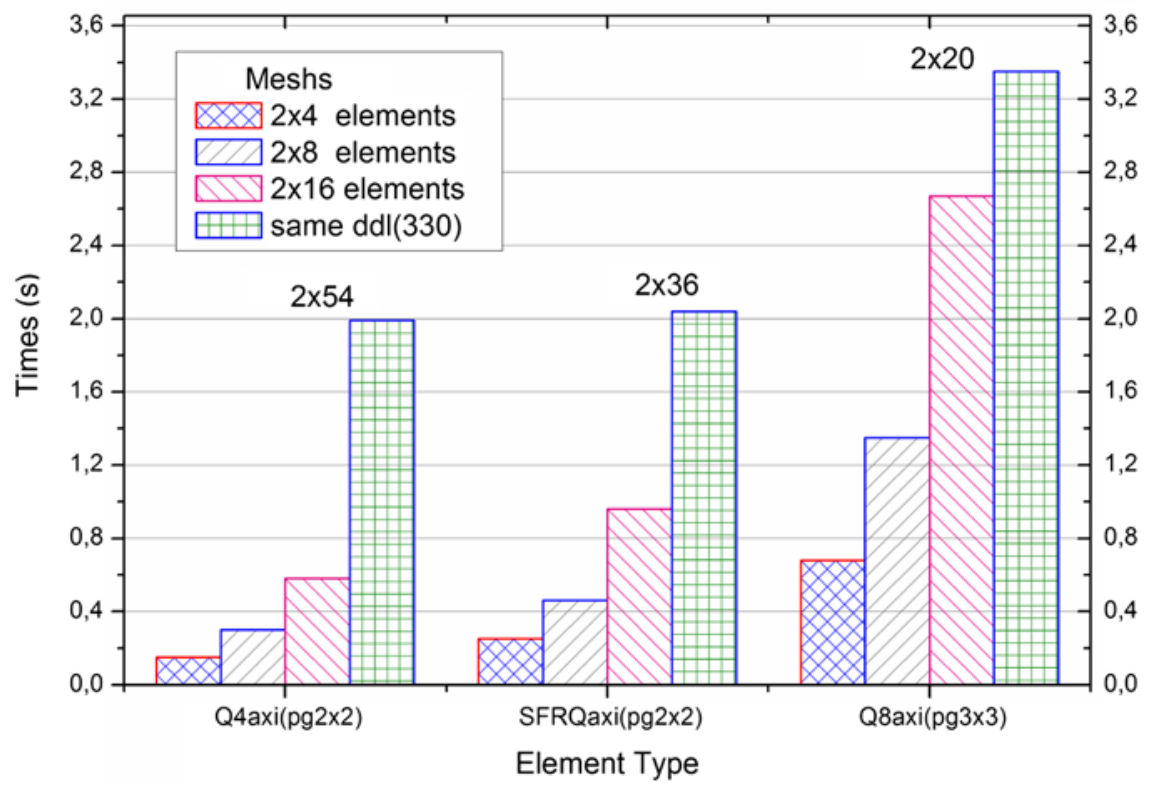

Figure 12. Elements performances - CPU time.

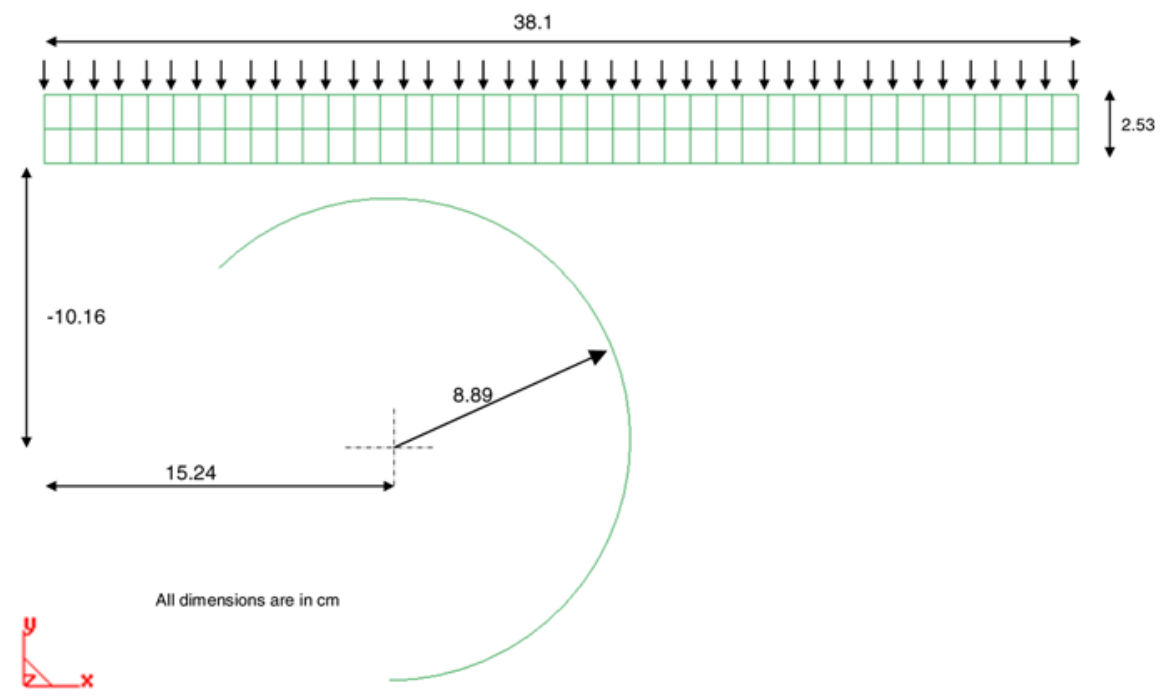

Figure 13. Inflection of a plate around a cylinder toric.

performs well similar to the Hughes solution with a small CPU time. On the other hand, Q4-Axi seems turning more quickly. It cannot give an accurate solution if the elements are distorted or the mesh is not sufficient.

\subsection{Hyperelastic circular plate around a cylinder toric}

Another test of a circular plate subjected to a pressure with constants of Mooney-Rivlin is $C_{1}=80 \mathrm{psi}(0.55 \mathrm{MPa}), C_{2}=20 \mathrm{psi}(0.14 \mathrm{MPa})$ and coefficient of penalty $\kappa=10^{5} \mathrm{Psi}$ 
$(68.9 \mathrm{MPa})$ (see Figure 13) is proposed by us to show the behaviour of the element under the effect of the inflection in Figure 13. The contact of the plate with the rigid body is supposed sticky. This test is solved in quasi-static using the Newton-Raphson method.

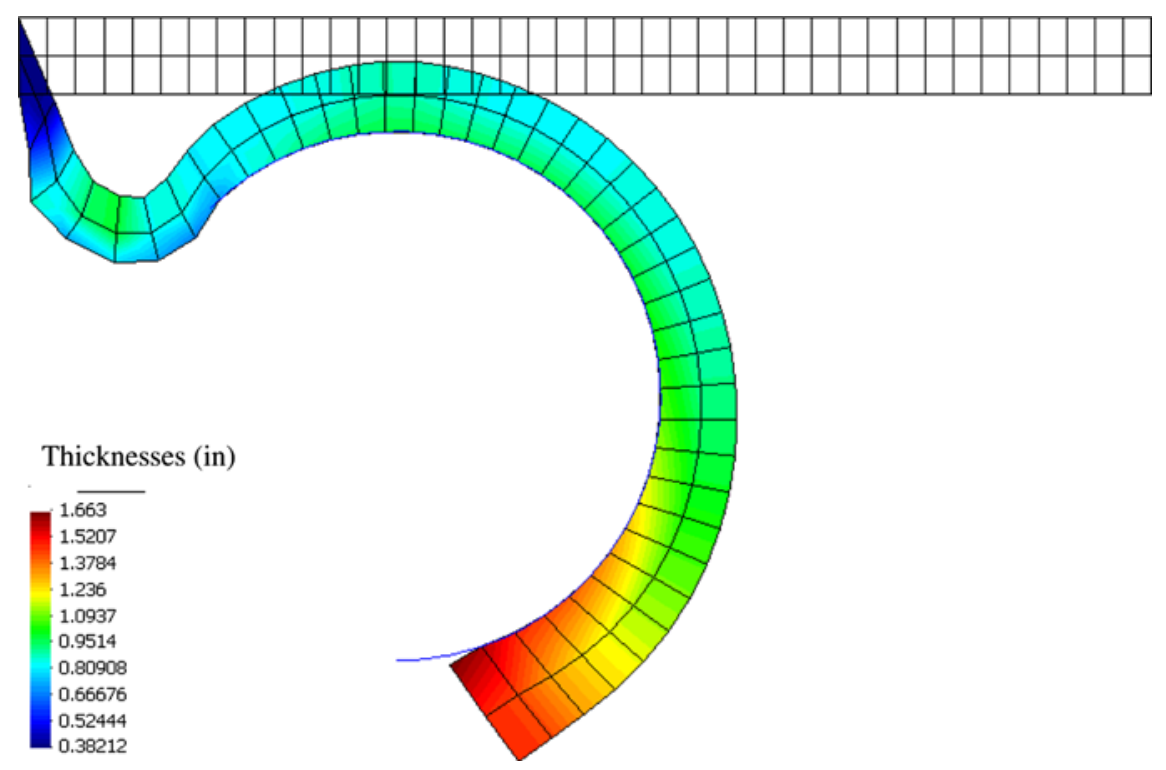

Figure 14. The circular plate shape after deformation.

(in)

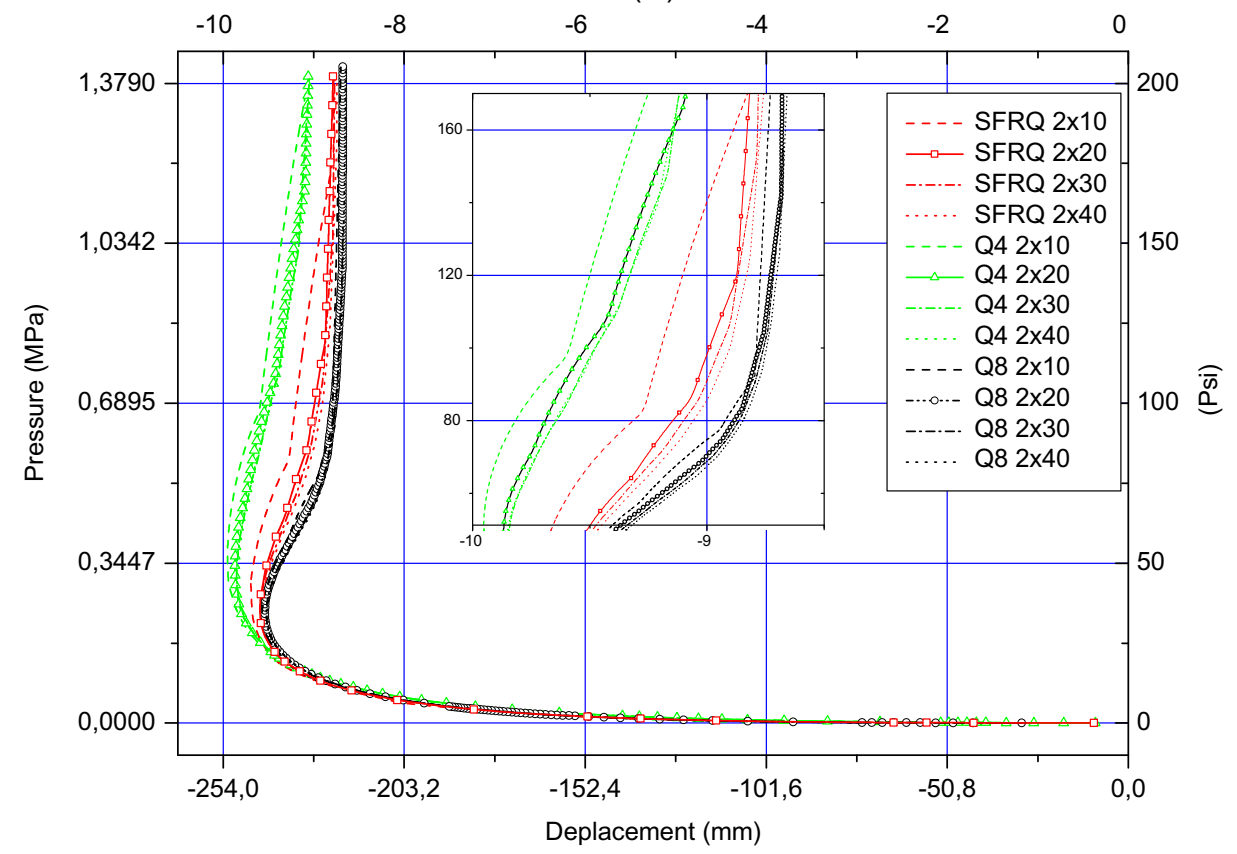

Figure 15. Loading curve for various meshes. 


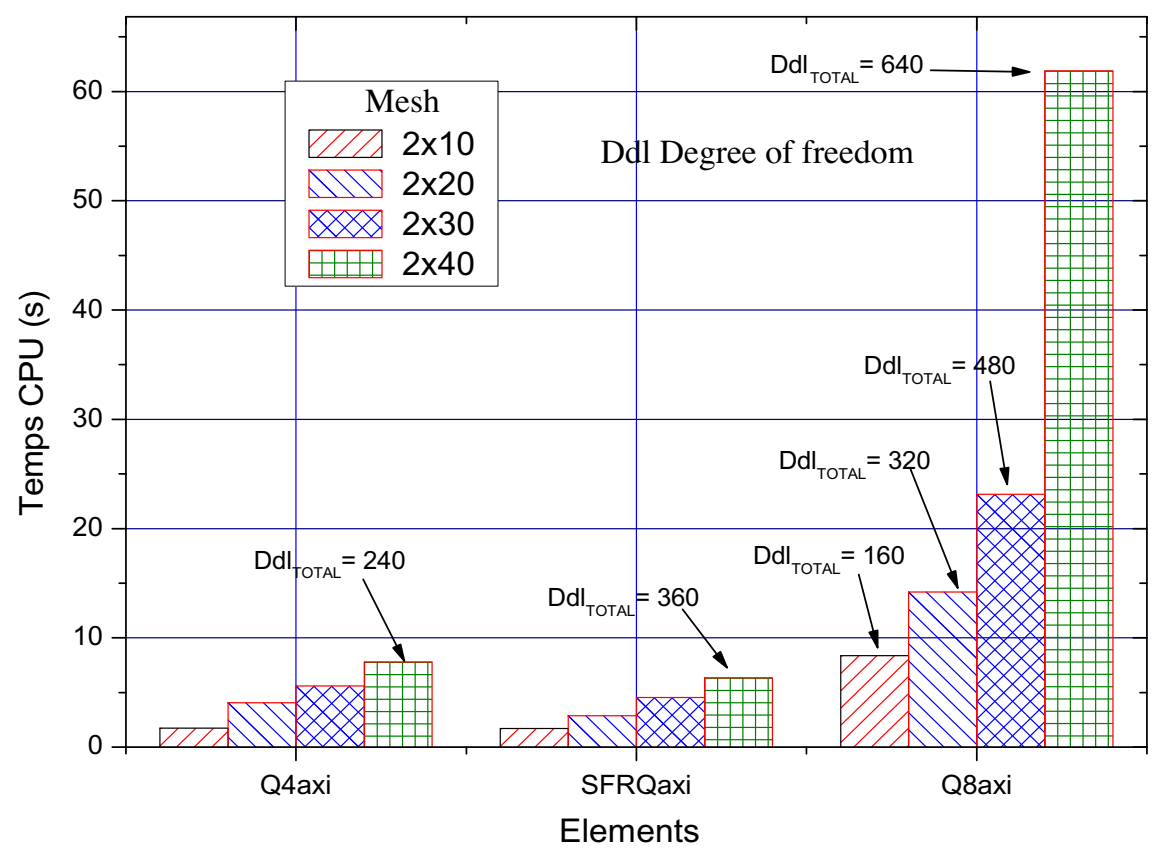

Figure 16. CPU time for various meshes.

The plate has to work in a formulation close to quasi-incompressible, which means we have a very low volume change. The plate does not stretch much, causing a thickness distribution in inches as shown in Figure 14. The plate subjected to a thinning has a 0.38 (in) where a difference of about 0.62 (in) and a thickening of 1-1.66 (in) with a difference of 0.66 (in), so what is lost to one side is won on the other side.

The SFRQ-Axi element is compared with elements Q4-Axi and Q8-Axi which is taken as a reference solution. Several sizes of meshes have been tested and compared. The results obtained for SFRQ-Axi (Figure 15) are close to Q8-Axi results for a mesh of $2 \times 20$ elements. These results remain much better than the Q4-Axi element even with a very refined mesh. The SFRQ-Axi has a very interesting rate of convergence in CPU time vs. Q4-Axi and Q8-Axi although the SFRQ-Axi element has an additional rotation of degree of freedom (Figure 16). The element SFRQ-Axi was tested for two cases with and without contact. For the first case, the element works in membrane and it can be an alternative for the type element Axi-Q8. The element SFRQ-Axi converges twice more rapidly for solving the same number of equations. For the second case, the behaviour of the element SFRQ-Axi with contact presence gives a very high rate of convergence. Because there are only two nodes in contact instead of three of the Axi-Q8 element. By considering two nodes per side reduces management candidate nodes on contact. Also the element SFRQ-Axi gives quite good accuracy which is similar to the element Axi-Q8.

\section{Conclusion}

The element developed gives results similar to that of Q8-Axi deemed effective with less CPU time by more than half for the same number of elements without contact, because the element is simply integrated with $2 \times 2$ Gauss points instead of $3 \times 3$, which has a major 
advantage in gain of CPU time, to address problems with non-linear hyperelastic laws. In the presence of contact, the element gives results very close to the time but with Q8-Axi CPU can be reduced to one-tenth for the same number of elements used. This advantage can be exploited to do simulations of formal forms of thermoformed products.

SFAQ-Axi can offer a good compromise between the execution speed and the required accuracy.

We note working in $3 \mathrm{D}$ it is necessary to have a penalty term of the energy density adequate to converge quickly and give the desired results. Unfortunately, in some simulations, the pure incompressibility leads to serious numerical difficulties.

\section{References}

Ayad, R. (2002). Contribution to the numerical modeling for solid and structure analysis, and for nonNewtonian fluid forming (HDR Thesis, University of Reims Champagne-Ardenne).

Crisfield, M.A. (1997). Non-linear finite element analysis of solids an structures (Vol. 2). England: Wiley.

Ghomari, T. (2007). Contribution to 3D modeling of plastic bodies forming ( $\mathrm{PhD}$ thesis, University of Reims Champagne-Ardenne).

Heinstein, M.W., Mello, F.J., Attaway, S.W., \& Laursen, T.A. (2000). Contact-impact modelling in explicit transient dynamics. Computer Methods in Applied Mechanics and Engineering, 187, 621640.

Hughes, T.J.R., \& Carnoy, E. (1983). Nonlinear finite element shell formulation accounting for large membrane strains. Computer Methods in Applied Mechanics and Engineering, 39, 69-82.

Mooney, M. (1940). A theory of large elastic deformation. Journal of Applied Physics, 6, 582-592.

Ogden, R.W. (1972). Large deformation isotropic elasticity-on the correlation of theory and experiment for incompressible rubber like solids. Proceedings of the Royal Society of London, Series A: Mathematical and Physical Sciences, 326, 565-584.

Rivlin, R.S. (1948). Large elastic deformation of isotropic materials. I. Fundamental concepts. Philosophical Transactions of the Royal Society of London, 240, 459-490.

Sze, K.Y., Zhenga, S.J., Lob, S.H. (2004). A stabilized eighteen-node solid element for hyperelastic analysis of shells. Finite Elements in Analysis and Design, 40, 319-340.

Treloar, L.R.G. (1976). The mechanics of rubber elasticity. Proceedings of the Royal Society of London, $351,301-330$.

Wang, S., \& Makinouchi, A. (2000). Contact search strategies for FEM simulation of the blow modelling process. International Journal for Numerical Methods in Engineering, 48, 501-521.

Williams, J.G. (1970). A method of calculation for thermoforming plastics sheets. Journal of Strain Analysis, 5(1), 49-57.

Zhong, Z.H., \& Nilsson, L. (1994). Automatic contact searching for dynamic finite element analysis. Computers \& Structures, 52(2), 187-197.

Ziane, M. (1999). Contribution à la simulation numérique du soufflage thermoformage de corps creux plastiques axisymétriques (Thèse de doctorat, Université de technologie de Compiègne).

Zouari, W., Ayad, A., Ben Zineb, T., \& Benjeddou, A. (2012). A piezoelectric 3D hexahedral curvilinear finite element based on the space fiber rotation concept. International Journal for Numerical Methods in Engineering, 90(1), 87-115. 